\title{
Central Nervous System Neoplasm
}

National Cancer Institute

\section{Source}

National Cancer Institute. Central Nervous System Neoplasm. NCI Thesaurus. Code C9293.

A benign or malignant, primary or metastatic neoplasm that affects the brain, meninges, or spinal cord. Representative examples of primary neoplasms include astrocytoma, oligodendrog lioma, ependymoma, and meningioma. Representative examples of metastatic neoplasms include carcinoma and leukemia. 